Soriano, Cebrián y Maqueda (2013) / Revista de Empresa Familiar, 3(2), 43-52.

www.revistadeempresafamiliar.uma.es

\title{
Empresas familiares en Cataluña: la importancia del protocolo
}

\author{
Family Business of Cataluña: the importance of the protocol \\ Juan Soriano Llobera $^{\mathrm{a}} \cdot$ Alfonso Cebrián Díaz $^{\mathrm{a}^{*}} \cdot$ Francisco Javier Maqueda Lafuente $^{\mathrm{b}}$ \\ ${ }^{a}$ Departamento de Organización de Empresas, Universitat Politécnica de Catalunya (Spain) \\ ${ }^{b}$ Departamento de Economía Financiera II. Comercialización e Investigación de Mercados, Universidad del País \\ Vasco (Spain))
}

\section{A T O S A R T ÍC U L O}

\section{Historial:}

Recibido 18-03-2013

Aceptado 15-09-2013

Palabras clave:

Empresa Familiar

Capacidades Innovadoras

Códigos JEL:

L1, M2

\section{R E S UMEN}

En el presente estudio vamos a realizar un análisis comparativo entre la Empresa Familiar y no Familiar en Cataluña.

La empresa que dispone de ventajas competitivas tiene mayor capacidad para competir activamente en el mercado, y ello es especialmente importante cuando la empresa interactúa en diferentes mercados exteriores.

Al final del presente estudio se exponen conclusiones y recomendaciones.

\section{A R T I C L E IN F O}

Article history:

Received 18-03-2013

Accepted 15-09-2013

Keywords:

Family Business

Innovative Capabilities

JEL codes:

\section{A B S T R A C T}

The objective of this research is to make a comparative study of familiar and non familiar business in Cataluña

The competitive advantage enables companies to be competitive in the markets, mostly when a company interacts with external markets.

Conclusions and recommendations are provided.

\section{L1, M2}

\footnotetext{
* Autor de contacto.

Correoselectrónicos: juan.manuel.soriano@upc.edu,acebrian@igema.net,javier.maqueda@ehu.es
} 


\section{Introducción}

Casi $\quad 400.000 \quad$ empresas, 391.676 exactamente, cesaron en todas sus operaciones durante el 2011 registrando la cifra de cierres más importante de los últimos 30 años. Contrariamente, $\quad 324.514 \quad$ compañías comenzaron el ejercicio de actividad económica en el mismo periodo. Ello arroja un saldo negativo próximo a las 60.000 empresas, según datos del informe económico Estructura y Demografía Empresarial publicado por el Instituto Nacional de Estadística (INE, 2012). El número de negocios activos en España se redujo un 1'7\% durante el año anterior y se situó en un total de 3.189.716 sociedades. Esta bajada supone el cuarto descenso anual consecutivo en el número de empresas en nuestro país (INE, 2012).

Las actividades económicas que más empresas crearon en términos netos entre el 1 de enero de 2011 y el 1 de enero del siguiente año fueron: educación, con 2.682; actividades sanitarias, con 1.478; actividades deportivas, recreativas y de entretenimiento, con 859; programación, consultoría y otros ejercicios relacionados con la informática, con 828; y actividades auxiliares a los servicios financieros y a los seguros, con 661 (Banco de España, 2012).

Por su parte, las actividades en las que más compañías cesaron en términos netos el pasado año fueron la construcción (-13.206), actividades de construcción especializada (10.170), servicios de arquitectura, ingeniería, ensayos y análisis técnicos (-7.343), así como otros servicios personales $(-5.261)$ y comercio al por menor, excepto de vehículos de motor y motocicletas (- 4.637) (Banco de España, 2012).

Pese a la contundencia de estos datos oficiales los grandes grupos autóctonos de capital familiar no sólo han logrado mantenerse a flote en la peor de las tormentas económicas de los últimos años, sino que en la mayoría de los casos han mantenido o incrementado su tamaño y preservado el grueso de los puestos laborales. Si nos referimos a Cataluña, territorio en el que las empresas familiares han tenido desde siempre una gran tradición, alrededor del
$70 \%$ de estos grupos mantienen relaciones internacionales, con presencia industrial $o$ comercial con países principalmente europeos, aunque como veremos, en algunos casos también con importante presencia en otros continentes.

Algunos de los ejemplos más conocidos y que son muy representativos de esta tendencia creciente son Mango, Roca, Freixenet, Codorniu, Grifols, Cirsa o Celsa.

\section{La Empresa familiar en un contexto de crisis económica}

Las empresas familiares son organizaciones sociales complejas, ya que en ella conviven la empresa y la familia (Díez, 2011). Como señalan De Querol, Barquero y Maqueda (2012) las investigaciones en la empresa familiar ha evolucionado en los últimos años, lo que puede ser apreciado en el aumento del volumen de trabajos publicados logrando abrirse camino como ámbito de estudio desde la Dirección de Empresas pudiendo considerarse como una disciplina científica joven (Chrisman et al., 2008). A pesar de ser un tema emergente en la investigación económica aún no existe una aceptada y generalizada definición del concepto de empresa familiar (Litz, 1995; Rus y Rodriguez, 2005; Miller et al., 2007; Rojo, Dieguez y Soto, 2011;Bañegil, Barroso y Tato, 2011; Cabrera Suárez (2012)).

La disciplina no ha tenido un gran desarrollo hasta la última década y la literatura no es tan voluminosa como en otras áreas de la Administración de Empresas (Bird et al., 2002; Benavides, Quintana y Guzmán, 2013).

Los temas tratados con mayor profusión son la sucesión (Lansberg \& Astrachan, 1994; Sharma, Chrisman, \& Chua, 2003), gobierno de la empresa familiar (Miller \& Le Breton-Miller, 2007) o dirección estratégica (Chrisman, Chua \& Sharma, 2005.

La crisis económica mundial ha incrementado notablemente el numero de procesos de concurso de acreedores y cierres de empresas en todo el mundo. Mas de treinta millones de empleos se han perdido en estos 
ultimo años por la crisis (IMF, 2010). Como señalan Casillas, Barbero y Moreno (2013) el fracaso supone un fenómeno importante dentro de la administración de empresas, especialmente en entornos hostiles o ante una crisis económica $\mathrm{y}$ financiera como la actual.

Según un reciente informe no publicado del Departament d'Organització d'Empreses de la Universitat Politècnica de Catalunya (UPC), en Cataluña hay un grupo de 13 empresas de capital familiar que han logrado superar ya la barrera de los 1.000 millones de euros de facturación. Otros tres grupos sobrepasan la barrera de los 2.000 millones. En cualquier caso el tamaño de estos conglomerados sigue siendo pequeño en comparación con el de otros negocios europeos de sagas familiares, principalmente italianos y franceses.

Si continuamos con el ejemplo de Cataluña, merece también tener en cuenta otro factor distintivo como es la amplia diversidad de sectores en los que operan estos grupos familiares, si continuamos con el ejemplo de Cataluña. Según el mismo estudio de la UPC, los sectores en los que se produce mayor actividad de este tipo de empresas familiares son: industria farmacéutica, construcción, sector editorial, gran distribución, alimentación $\mathrm{y}$ metal (UPC, 2012).

De esta manera, grupos tan conocidos como Puig, Agrolimen, Nutrexpa, Ferrer, Almirall, Esteve, Planeta y otros, han destacado en los últimos años tanto por su pujante posición en sus respectivos sectores como por su internacionalización.

A este conjunto de negocios familiares de reconocida tradición y notoriedad se han unido en los últimos años otras empresas que han ido escalando puestos de mérito en el ranking de los mayores grupos de tradición familiar: ComsaEmte, las citadas anteriormente Grifols y Mango, Casa Tarradellas, Tous, Ros Roca, Quadis, Desigual, Bon Preu y La Farga. Observamos, pues, que las empresas de alimentación, distribución y construcción tienen un notable peso en el listado de los grandes grupos familiares catalanes.
Esta circunstancia de la empresa familiar española, haber mantenido o aumentado su negocio desde el inicio de la crisis, no es exclusivo de nuestro país. Según la encuesta mundial de la empresa familiar 2012 (PwC, 2012), elaborada por PwC entre casi 2.000 empresarios de 30 países, estos grupos resisten mejor la crisis que otras compañías y tienen una capacidad mayor que el resto del tejido económico para captar las oportunidades que genera de la actual situación. Y lo más significativo es que, según este estudio, el $80 \%$ prevé que sus ventas sigan creciendo como lo han hecho hasta ahora $\mathrm{o}$, incluso, a un ritmo mayor en los próximos ejercicios.

Todo este escenario se produce como consecuencia de las propias características que tienen los grupos familiares. Una actitud positiva, inversiones más a largo plazo que el resto de las empresas, más atentas a maximizar los beneficios en el corto plazo.

Pero lo fundamental del éxito que ha permitido mantener esta situación ha sido principalmente presentar unas finanzas saneadas de que ha posibilitado afrontar sin más traumas de los debidos el desplome del consumo interior. Además la situación también ha sido propicia para acometer oportunidades de compra para reformar el tamaño de la empresa familiar y, a la vez, diversificar su actividad.

En los años de bonanza económica las empresas familiares tenían difícil acceder a determinadas operaciones de compra pues su carácter industrial y su visión a largo plazo del negocio les impedía pagar los elevados múltiplos que estaban dispuestos a satisfacer el capital riesgo o compañías con un claro perfil financiero. La crisis ha tenido una consecuencia clara si atendemos a los datos objetivos: el triunfo de la empresa familiar frente al inversor especulador que ha pululado por España a sus anchas, especialmente en la primera parte de la década de este siglo.

En tiempos difíciles el capital riesgo "huye" de los escenarios turbulentos pues ve difícil materializar sus plusvalías entre los 5 y 10 años desde la inversión, como ha sido costumbre. No hay que olvidar que la legislación tributaría 
incentivaba estas situaciones. El concepto de empresario ha quedado, debido a la crisis, diametralmente definido en contraposición con el concepto de inversor temporal, la mayor parte de las veces equiparable a especulador.

Otra característica de la empresa familiar es su necesidad limitada, por los argumentos expuestos anteriormente, de recurrir necesariamente al crédito bancario. En un momento en el que los bancos no cumplen su principal misión histórica como es conceder crédito, la solidez financiera de las compañías juega un papel tan importante pues puede acabar con la historia de la empresa.

\section{El papel de los bancos en la crisis económica}

Los bancos no otorgan crédito porque tienen en su balance un excesivo número de préstamos y comparativamente un escaso volumen de depósitos. Además, a causa de los excesos cometidos en el pasado, más de uno tiene problemas de solvencia, como se ha constatado en los últimos meses tras los test realizados a instancias de BCE. La situación actual es consecuencia de los errores cometidos por los directivos y el Banco de España y también de las injerencias políticas. Los principales errores provienen de creer que el precio de la vivienda en España no podía bajar.

Este convencimiento hizo que las entidades concedieran excesivos créditos, tanto a promotores como a compradores de la vivienda. Afortunadamente la visión empresarial de los grupos familiares se mantuvo alejada del escenario inmobiliario que supuso uno de los principales motores económicos durante casi dos décadas.

También influyó el pensamiento respecto a que a la banca española no le afectaría la crisis financiera internacional, una idea que nació por la inexistencia de hipotecas subprime de los EEUU en los balances de los bancos españoles, la creación de provisiones anticíclicas durante la etapa de bonanza y de las elevadas penalizaciones establecidas por el Banco de España a las entidades que tragasen activos fuera del balance. Además, pensar que la crisis económica sería corta también hizo daño. Si hubiera sido así, el agujero general por la bajada del precio de los activos inmobiliarios se habría compensado con la subida posterior.

Pero la crisis se ha alargado y la están sufriendo en mayor medida los que apostaron por sectores de alta rentabilidad a corto y con buena dosis de especulación.

A todo ello hay que sumar las fusiones de carácter político entre cajas de ahorros, que fueron las que dieron mayor número de préstamos inmobiliarios $\mathrm{y}$, en consecuencia, presentan actualmente la tasa más alta de morosidad. Las entidades que se unían eran principalmente de las mismas comunidades autónomas o gobernadas por el mismo partido político. Esta estrategia provocó que una caja que iba mal se acabase uniendo a otra que todavía estaba peor. Y se añadió la ocultación de las pérdidas en que se había incurrido.

Con el visto bueno del Banco de España, numerosas entidades valoraron sus activos por su valor histórico o hipotecario. De esta forma podían continuar presentando beneficios anuales, ya que reducían considerablemente el efecto sobre sus cuentas de la explosión de la burbuja. Espero que el gobierno sea consciente de los numerosos errores cometidos en el pasado y no hagan ninguno más. La economía española necesita ya una definitiva reforma financiera que sanee las entidades para que vuelvan a conceder crédito. Tradicionalmente la empresa familiar ha sido más "amiga" de los bancos que de las cajas. No porque se les supusiera un futuro negro, que ha desembocado en la desaparición de la mayoría de ellas, sino por ser más ágiles en el negocio bancario.

Sin embargo la actual recesión también ha paralizado algunas salidas al mercado bursátil de compañías familiares que habían previsto acceder al parqué bien a través del mercado continuo o bien del mercado alternativo bursátil (MAB), en función de su tamaño y estructura. Incluso alguna de ellas como Mecalux ha tenido una corta presencia en la bolsa tras haber provocado su propia exclusión. 


\section{Las empresas familiares catalanas}

Con datos al finalizar el año 2012 sólo seis de las 50 mayores empresas familiares catalanas cotizan en la bolsa española. Se trata de Almirall, Damm, Fluidra, Grifols, Catalana Occidente y Cementos Molins (Bolsa de valores de Barcelona, 2012).

Todas estas compañías mantienen una cotización estable dentro de los vaivenes bursátiles a los que nos tiene acostumbrados las cifras negativas de la economía española en los últimos dos años, principalmente. Incluso alguna de estas empresas cotizadas presenta cifras de evolución favorables notablemente superiores a la media.

Todas estas reflexiones tienen un punto en común. La histórica aversión al riesgo que caracteriza a los gestores de las empresas familiares, algo que había sido criticado en tiempos pasados, se ha demostrado que era una de sus mejores bazas. El inicio de la crisis pilló a muchas empresas con balances poco capitalizados y fuertes endeudamientos. Precisamente todo lo contrario de lo que en general sucedió con los grupos empresariales de carácter familiar.

El entorno y la velocidad con la que en los últimos tiempos han cambiado grandes modelos de negocio, han afectado notablemente al éxito de la empresa. La volatilidad de los mercados y la complejidad de las propias organizaciones llegan a generar tensión en capas directivas. También en este caso estos cambios como consecuencia de los mercados han sido claramente más contenidos en las empresas familiares, debido principalmente a que la estructura directiva acostumbra a tener unas connotaciones diferentes a lo que podríamos entender como aquellas convencionales.

Un informe de la consultora GD\&A Business Brokers (GD\&A Business Brokers, 2011) realizado en 2011 en Cataluña sobre 31.711 pymes, señala que la permanencia de directivos en las empresas de carácter familiar es notablemente superior que en negocios de otras estructuras. Según el citado informe esta fidelidad en el puesto de trabajo supone una

media de un $32 \%$ superior en los grupos familiares.

El mismo trabajo aporta otros datos de interés en relación a las pymes catalanas y, en este caso, $\sin$ referirse específicamente a la estructura familiar. Un $60{ }^{\prime} 3 \%$ de las empresas catalanas que facturan hasta 30 millones de euros al año, presentan un endeudamiento superior al $60 \%$ de su activo, lo que compromete seriamente su futura viabilidad. Esta situación - dice el informe - afecta a 19.121 empresas catalanas sobre un total de 31.711 (excluidas las promotoras e inmobiliarias) que constituyen el censo global de compañías de esta dimensión, que es el más numeroso de cuantas existen en Cataluña. Con este dato se puede deducir que el grado de infracapitalización es especialmente significativo en la actual coyuntura de amplia restricción crediticia.

Otra aportación de interés de este trabajo de la consultora GD\&A Business Brokers se refiere a la antigüedad de las compañías analizadas. El 18\% de ellas (de la muestra de 31.711) se constituyeron hace 25 años o más, por lo que han debido o deben abordar la sucesión.

Finalmente este trabajo se refiere al interés de posibles operaciones de compra-venta o fusión de estas empresas analizadas para adquirir mayor masa crítica. Al $76 \%$ de los empresarios encuestados "no les interesa en absoluto" o ve "poco probable" la entrada de un nuevo accionista en su compañía. Sólo un $26 \%$ afirma haber participado en alguna operación de compra de otra compañía "por razones de mercado". Sin embargo, y esto conviene destacarlo, la totalidad de estos empresarios manifiesta que está "satisfecho o muy satisfecho" por la transacción realizada, que ha cubierto sus expectativas. No hay que olvidar que las pymes catalanas representan algo más del $95 \%$ del total de empresas radicadas en Cataluña, y aglutinan el 75'2\% del empleo. Ello pone de manifiesto un tejido empresarial muy atomizado.

\section{El protocolo en la empresa familiar}

Soriano Llobera, J., Cebrián Díaz y Maqueda Lafuente, F.J. (2013). Empresas familiares en Cataluña: la importancia del protocolo. Revista de Empresa Familiar, 3(1), 43-52. 
Otro aspecto especialmente significativo y de carácter eminentemente jurídico aunque de trascendencia económica, es el protocolo en la empresa familiar que ha demostrado su eficacia como instrumento diseñado para evitar disputas en las empresas familiares. Esta norma, que se ha ido completando a lo largo de los años desde que en 2003 se previó en la Ley de Sociedad Limitada Nueva Empresa. En 2007 apareció por primera vez en un marco legal, que afecta a este tipo de empresas, la figura del Consejo de Familia. Se reconoció su papel como instrumento regulador de la dinámica de la familia propietaria y abrió la vía para que en el futuro tuviera valor legal y que sus decisiones fueran vinculantes, tal como sucede con los consejos de administración.

Nebreda (2010) enfatiza la importancia del protocolo familiar y en especial de su proceso de elaboración, porque salen a la luz todos los temas clave. Temas que, por otra parte, pueden afectar de forma significativa y de lleno al futuro de una empresa como normas y condiciones de acceso (por parte, por ejemplo, de la familia política), transmisión de las participaciones, regímenes económicos matrimoniales, usufructos (mantenimiento de vienes hasta que el interesado pueda hacerse cargo, en ese caso la empresa) o la entrada de directivos y ejecutivos no vinculados a la familia propietaria en la empresa.

Blanco-Morales (2007) afirma que "dar a conocer el protocolo con las garantías que ofrece el Registro Mercantil, tiene como ventajas transmitir una imagen de solvencia, estabilidad y garantía de continuidad de la empresa".

Algo muy importante en unas empresas atenazadas en ese momento por problemáticas particulares, en especial la sucesión, la continuidad del negocio y los conflictos familiares.

Si nos referíamos al inicio de este artículo a la dimensión de las empresas familiares en España, y especialmente en Cataluña donde tradicionalmente han tenido gran proyección, es evidente que las micropymes ocupan un lugar muy destacado en cuanto a su número. Por tal motivo todo el proceso que conlleva la elaboración de un protocolo completo, de hecho ha frenado la creación de algunos. Además de exigir una, en ocasiones no fácil, negociación con los integrantes de la familia. Por todas estas razones y alguna más, los protocolos completos sólo están presentes en no más de un $15 \%$ del total de empresas familiares.

La forma de llevar a cabo el proceso de valoración de una empresa familiar dista en algunos aspectos de las que no tienen ese carácter. Sin embargo es un proceso cada día más importante y básico. El incremento de los distintos negocios jurídicos que se realizan entre las empresas, y entre éstas y otros agentes económicos, hacen necesaria cada vez más este tipo de actuaciones con finalidad de valoración objetiva. Es precisamente en épocas de incertidumbre y de dificultad en la predicción y previsión de los acontecimientos económicos futuros, cuando se hace más necesario resaltar el concepto de valor y los procesos que ayudan de forma objetiva a determinar ese valor. En todo proceso de valoración de una empresa es necesaria una primera reflexión: ¿para qué? y ¿para quién? Es decir, cuál es la finalidad de ésta. La respuesta a estas preguntas nos lleva a conocer los motivos y las múltiples finalidades que tanto agentes internos, como propietarios o accionistas, y externos, como inversores o entidades financieras, se posicionan como posibles demandantes a este valor.

A lo largo de la vida de una empresa, son muchos los motivos que originan la necesidad de ser valorada: operaciones de compraventa, ampliaciones de capital con entrada de nuevos socios e inversiones; salidas a bolsa o valoración de empresas que se encuentran en proceso de expansión o bien por motivo de herencias, testamentos y protocolos familiares, así como disolución de sociedades conyugales; daciones en pago de deuda, procesos de reestructuración de deuda y obtención de líneas de financiación; concurso de acreedores, y un largo etcétera. Para esta valoración empresarial serán elementos de especial significación los habituales atributos a los que nos referíamos anteriormente como es la capitalización de las compañías familiares, su menor dependencia del 
crédito bancario en consecuencia y la internacionalización de buena parte de ellas, de la que nos referiremos más adelante.

En definitiva de la valoración de una empresa es un proceso por el que se busca la cuantificación de los elementos que constituyen su patrimonio, su actividad, su potencialidad o cualquier otra característica intrínseca de ésta, susceptible de ser analizada y a continuación valorada.

El fundador de una empresa sabe que la elección de su heredero es un hecho que llegará de forma ineludible, $\mathrm{y}$ de esta decisión dependerá la marcha del negocio: se traspasa el patrimonio, pero no se hereda el cargo.

Tàpies (2011) pone como ejemplo que a nadie se le ocurra dar por hecho que el hijo de un gran violinista será también un virtuoso de ese instrumento musical. Pero, en cambio, se da por sentado que los descendientes de un gran empresario heredarán su gen emprendedor. En cualquier planificación de un proceso de sucesión se deben establecer los criterios del perfil idóneo del sucesor en función de las necesidades del negocio, así como la toma de decisión, su comunicación y el periodo de transición.

Según la opinión de José Téllez, socio senior

Tabla 1.

Principales grupos familiares catalanes en 2011

PRINCIPALES GRUPOS FAMILIARES CATALANES

\begin{tabular}{|c|c|c|c|}
\hline Grupo & Sector & $\begin{array}{l}\text { Ventas } 2011 \\
\text { (millones } € \text { ) }\end{array}$ & Familia propietaria \\
\hline Celsa & Siderurgia & 4.806 & Rubiralta \\
\hline Planeta & Editorial & 3.182 & Lara \\
\hline Comsa Emte & Infraestructura & 2.159 & Miarnau y Sumarroca \\
\hline Nortia (Cirsa) & Juegos y ocio & 1.870 & Lao \\
\hline Grifols & Farmacéutico & 1.795 & Grífols \\
\hline Catalana Occidente & Seguros & 1.780 & Serra \\
\hline Cobega & Bebidas & 1.600 & Daurella \\
\hline Agroaliment & Alimentación & 1.577 & Carulla \\
\hline Roca Corporación & Sanitarios & 1.551 & Roca \\
\hline Mango & Moda & 1.408 & Andic \\
\hline Puig & Moda y perfumes & 1.343 & Puig \\
\hline La Farga Group & Metalúrgica & 1.300 & Fisas y Guixà \\
\hline Bamesa & Metalúrgica & 1.000 & Arasa \\
\hline Petromiralles & Gasolinera & 982 & Torrens \\
\hline Ficosa & Comp. Automoción & 973 & Pujol y Tarragó \\
\hline Werfen & Material hospitalario & 970 & Rubiralta \\
\hline Vall Companys & Ganadero & 950 & Vall Palou \\
\hline Miquel Alimentación & Alimentación & 920 & Miquel \\
\hline Molins & Cementero & 830 & Molins \\
\hline Esteve & Farmacéutico & 814 & Esteve \\
\hline Damm & Bebidas & 806 & Carceller, Bosch, Agenjo y Furriol \\
\hline Condis & Supermercado & 806 & Condal \\
\hline Ferrer & Farmacéutico & 803 & Ferrer Salt \\
\hline Almirall & Farmacéutico & 768 & Gallardo \\
\hline Indukern & Químico-farmacéutico & 761 & Díaz-Varela \\
\hline
\end{tabular}


de Legal de la consultora internacional Ernest \& Young (Elías, 2012). la prudencia tradicional en la empresa familiar, que ha sido un factor fundamental para sobreponerse a la crisis que se inició en 2007, pero no menos importante ha sido la apuesta que en su día hicieron las empresas familiares por el mercado exterior.

Gracias a ello, muchas empresas familiares han visto como la demanda internacional "tiraba" del negocio frente a un mercado interior castigado por la crisis de consumo. Por citar un ejemplo: el del fabricante de sanitarios Roca que el pasado año registró ya más ventas en Brasil que en España. Concretamente facturó 309,5 millones de euros en ese país de la América Latina - donde tiene 3.200 trabajadores -, un $20 \%$ del total. Otras compañías como Puig Beauty and Fashion se encarama al grupo del top 5 de la perfumería selectiva mundial, y su negocio exterior aporta casi el $80 \%$ del total (Puyol, 2012).

La tabla 1 nos muestra las 25 principales empresas familiares en función de las ventas registradas en 2011 con especificación del sector de actividad al que pertenecen y, en la última columna, la familia propietaria o principal accionista de ésta. No pasa desapercibido el hecho que tan sólo seis de estas 25 empresas registre menos ventas respecto al ejercicio anterior (2010) y, además, en porcentajes de descenso poco significativos. Ningún otro segmento de compañías puede lucir unas cifras de incremento similares.

Fuera de este ranking quedan otras compañías familiares de alta reputación y comercialmente conocidas como Casa Tarradellas, MRW, Freixenet, Nutrexpa, Tous, Soler\&Palau, Copcisa, Europastry-Fripan, Grupo RBA, Disbesa Darnés, entre otras.

\section{Conclusiones}

Al igual que en estudios anteriores (Fuentes, Hernández y Vallejo, 2008) hemos detectado que las empresas familiares Catalanas tienen como objetivo primordial el crecimiento y la sucesión de la empresa. Para ello es importante la consideración del protocolo.
El protocolo de sucesión de la Empresa Familiar debe de posibilitar que el sucesor sea el más cualificado, ya que existen estudios que destacan la importancia de la formación del gerente de la empresa (Martin y Staines, 1994; Carrasco y Meroño, 2011).

También se puede concluir que si bien es recomendable que la sucesión en la Empresa Familiar se haga contando con los miembros de la familiar, está no debe cerrarse a la posibilidad de que alguien externo a la familia dirija la empresa (casado, 2005), pudiendo solucionar los conflictos empresa-familiar ya que los accionistas tienden a defender la cuenta de resultados y los gerentes pueden perseguir de forma más específica el crecimiento y la continuidad empresarial. Los conflictos pueden generarse cuando los propietarios adoptan distintas posturas ante el reparto de beneficios (Llorente, 2012).

El gran desafío de las empresas actuales es pensar en el largo plazo y programar el futuro, evitando centrarse sólo en la gestión de las urgencias derivadas de la crisis. En esta etapa es especialmente importante el aspecto de la gestión. El manejo de los riesgos que derivan de un proceso de expansión, sea nacional o internacional, requiere ejecutivos expertos que, si no se encuentran en el seno de la familia, deberán buscarse en el mercado tras un serio y riguroso proceso para asegurar que el gestor va a compartir el grueso de la filosofía de los grupos familiares.

\section{Bibliografía}

Banco de España (2012, septiembre). Informe de Coyuntura, Madrid: Banco de España.

Bañegil Palacios, T.M., Barroso Martínez, A. y Tato Jiménez, J.L. (2011). Profesionalizarse, emprender y aliarse para que la empresa familiar continúe. Revista de Empresa Familiar, 1(2), 27-41.

Benavides-Velasco, C.A., Quintana-García, C., Guzmán-Parra, V.F. (2013). Small Business Economics 40 (1), 41-57.

Bird, B., Welsch, H., Astrachan, J.H. \& Pistrui, D. (2002). Family business research: The evolution of an academic field. Family Business Review, 15(4), 337-350. 
Bolsa de Valores de Barcelona (2012 diciembre). Boletín de cotización Bolsa de Valores de Barcelona, Barcelona: Bolsa de Valores de Barcelona.

Cabrera Suárez, M.K., (2012). La influencia de la familia en la empresa familiar: objetivos socioemocionales, stewardship y familiness. Revista de Empresa Familiar, 2(2), 93-96.

Carrasco Hernández, A. J. y Meroño Cerdan, A. L. (2011). Efectos de la formación universitaria del gerente de pymes familiares en la motivación laboral del empleado. Revista de Empresa Familiar, 1(1), pag. 35-51.

Casado, Juan, F. (2005) ¿Liderazo empresarial o liderazgo familiar? en J.F. Corona Ramón (coord.). Manual de la empresa familiar (pp. 33). Barcelona: Ediciones Deusto

Casillas Bueno, J.C., Barbero Navarro, J.L. y Moreno Menéndez, A.N. (2013). Reestructuración y tipo de propiedad en empresas en crisis. Diferencias entre empresas familiar y no familiares. Revista de Empresa Familiar, 3(1), 7-20.

Chrisman, J.J., Chua, J.H., Kellermanns, F.W., Matherne, C.F. III \& Debicki, B.J. (2008). Management journals as venues for publication of Family Business Research. Entrepreneurship Theory and Practice, 32(5), 927-934.

Chrisman, J.J., Chua, J.H. \& Sharma, P. (2003).

Current trends and future directions in family business management studies: Toward a theory of the family firm. Coleman White Paper Series, Madison, WI: Coleman Foundation and U.S. Association of Small Business and Entrepreneurship.

De Querol, N., Barquero Cabrero, M. y Maqueda Lafuente, J. (2012). Importancia de la estacionalidad y del conocimiento de la demanda turística para las empresas familiares españolas. Revista de Empresa Familiar, 2(1), 33-44.

Díez Soto, C. M. (2011). El pago de las legítimas en dinero: un instrumento para planificar la sucesión en la Empresa Familiar. Revista de Empresa Familiar, 1(1), 23-33.

Elías, B. (2012, 9 de noviembre). Cuando el heredero no sirve como directivo, Expansión. (http://www.expansion.com/2012/11/09/empleo/des arrollo-de-carrera/1352487955.html)

Fuentes Lombardo, G, Hernández Ortiz, M.J. y Vallejo Martos, M.C. (2008). Razones para crecer en la empresa familiar: un análisis compartivo. Investigaciones Europeas de Dirección y Economía de la Empresa, 14(3), 1534.

GD\&A Business Brokers (2011). Informe sobre pymes catalanas, Barcelona: GD\&A Business Brokers.

INE (2012) Informe Estructura y Demografia Empresarial del Directorio Central de Empresas (DIRCE) del Instituto Nacional de Estadística

International Monetary Found. (2010) Oslo Conference.

Lansberg, I. \& Astrachan, J.H. (1994). Influence of family relationships on succession planning and training: The importance of mediating factors. Family Business Review, 7(1), 39-59.

Litz, R.A. (1995). The family business: Toward a definitional clarity. Academy of Management Journal Best Papers Proceedings, 100-104.

Llorente Muñoz, V. (2012). Política de dividendos y conflicto en la empresa familiar: el protocolo. Revista de Empresa Familiar, 2(1), 5563.

Martin, G. y Staines. H. (1994). Managerial Competences in Small Firms, The Journal of Management Development, 12(7), .23-34.

Miller, D. \& Le Breton-Miller, I. (2007). Kicking the habit: Broadening our horizons by studying family businesses. Journal of Management Inquiry, 16(1), 27-30.

Nebreda, R. (2010, febrero). Garrigues aboga por ajustar la retribución de la empresa familiar al Buen Gobierno de las cotizadas, El Economista, 18. (http://www.eleconomista.es/interstitial/volver/Carm ignac nov-

dic13/economia/noticias/1920621/02/10/Economia-

Empresas-Garrigues-aboga-por-ajustar-laretribucion-de-la-empresa-familiar-al-BuenGobierno-de-las-cotizadas-.html)

PwC (2012, octubre). Encuesta mundial de la empresa familiar, Madrid: PwC.

Rojo Ramírez, A.A., Diéguez Soto, J. y López Delgado, P. (2011). Importancia del concepto de Empresa Familiar en investigación: utilización de la base de datos SABI para su clasificación. Revista de Empresa Familiar, 1(1), pag. 53-67.

Rus Rufino, S., Rodriquez Fernández, M.P. (2005) ¿Qué es una empresa familiar? en J.F. 
Corona Ramón (coord.). Manual de la empresa familiar (pp. 33). Barcelona: Ediciones Deusto

Sharma, P., Chrisman, J.J., \& Chua, J.H. (2003). Predictors of satisfaction with the succession process in family firms. Journal of Business Venturing, 18(5), 667-687.

Tàpies, J. (2011). Empresa familiar: ni tan pequeña, ni tan joven. Barcelona: Fundación Jesús Serra. 